\section{Mad2 prevents aneuploidy and premature proteolysis of cyclin $B$ and securin during meiosis $I$ in mouse oocytes}

\author{
Hayden A. Homer, ${ }^{1,2,5}$ Alex McDougall, ${ }^{2,4}$ \\ Mark Levasseur, ${ }^{2}$ Katie Yallop, ${ }^{1}$ \\ Alison P. Murdoch, ${ }^{1,3}$ and Mary Herbert ${ }^{1,3,6}$
}

${ }^{1}$ Newcastle Fertility Centre at Life, Centre for Life, Newcastle upon Tyne NE1 4EP, United Kingdom; ${ }^{2}$ School of Cell and Molecular Biosciences and ${ }^{3}$ School of Surgical and

Reproductive Sciences, The Medical School, University of Newcastle, Newcastle upon Tyne NE2 4HH, United Kingdom

In mitosis, the spindle checkpoint protein Mad2 averts aneuploidy by delaying anaphase onset until chromosomes align. Here we show that depletion of Mad2 in meiosis I mouse oocytes induced an increased incidence of aneuploidy. Proteolysis of cyclin B and securin commenced earlier in Mad2-depleted oocytes, resulting in a shortened duration of meiosis I. Furthermore, overexpression of Mad2 inhibited homolog disjunction. We conclude that Mad2 delays the onset of cyclin B and securin degradation and averts aneuploidy during meiosis I in mammalian oocytes. The data suggest a link between trisomies such as Down syndrome and defective oocyte spindle checkpoint function.

Supplemental material is available at http://www.genesdev.org.

Received April 16, 2004; revised version accepted October 14, 2004.

During meiosis, one round of DNA synthesis is followed by the sequential segregation of homologous chromosomes at meiosis I and sister chromatids at meiosis II. Although female meiosis I errors are the major genetic cause of miscarriage and mental retardation in humans, little is known about their molecular origins (Hassold and Hunt 2001). The spindle checkpoint is the principal mechanism for preventing chromosome missegregation during mitosis by delaying anaphase until completion of chromosome alignment (Mussachio and Hardwick 2002). Checkpoint proteins such as Mad2 prevent activation of a multi-subunit ubiquitin ligase called the anaphase-promoting complex or cyclosome (APC/C) by sequestering the APC/C activator protein, Cdc20 (Peters 2002). Once chromosomes establish a stable bipolar orientation on the mitotic spindle, the APC/C directs pro-

[Keywords: Aneuploidy; Mad2; spindle checkpoint; mouse oocytes; cyclin $\mathrm{B}_{\text {; securin] }}$

${ }^{4}$ Present address: UMR 7009 CNRS/Université Pierre et Marie Curie (Paris VI), Observetoire Océanologique, 06230 Villefranche-sur-Mer, France.

Corresponding authors.

${ }^{5}$ E-MAIL h.a.homer@ncl.ac.uk; FAX 44-191-219-4747.

${ }^{6} \mathrm{E}-\mathrm{MAIL}$ mary.herbert@ncl.ac.uk; FAX 44-191-219-4747.

Article and publication are at http://www.genesdev.org/cgi/doi/10.1101/ gad.328105. teolysis of securin and cyclin $\mathrm{B}$, thereby inducing anaphase and cytokinesis (Peters 2002). As in mitosis, securin and cyclin B destruction are required for homolog disjunction and exit from meiosis I in mouse oocytes (Herbert et al. 2003).

In mammalian somatic cells, the Mad2-dependent spindle checkpoint is required for mitotic arrest in the presence of spindle poisons (Li and Benezra 1996) and to delay anaphase until proper chromosome alignment in the absence of spindle damage (Gorbsky et al. 1998). During meiosis I in budding yeast, Mad2 is also required to arrest cells following spindle disruption and to avert nondisjunction in unperturbed cells (Shonn et al. 2000, 2003). In mouse oocytes, a dominant-negative variant of human Mad2 (hMad2) destabilizes cyclin-dependent kinase 1 (Cdk1) activity in the presence of the spindle poison nocodazole, indicating that Mad2 is required for meiosis I arrest in the face of spindle damage (Wassmann et al. 2003). It is not known, however, whether Mad2 regulates progression through meiosis I in unperturbed oocytes.

Conventional genetic approaches have not been informative regarding checkpoint function in female meiosis. For example, deletion of Mad2 and Bub3 causes mouse embryonic lethality (Dobles et al. 2000; Kalitsis et al. 2000). Furthermore, using such approaches, it would be difficult to distinguish between errors that occurred during premeiotic germ cell proliferation and those that occurred during meiosis I. To overcome this problem, we have used morpholino-based gene silencing to deplete Mad2 in fully grown oocytes, enabling us to determine the effect of Mad2 depletion specifically during meiosis I. We found that Mad2 is required to delay exit from meiosis I by preventing premature proteolysis of cyclin B and securin. Furthermore, Mad2 depletion resulted in an increased incidence of segregation errors during anaphase of meiosis I.

\section{Results and Discussion}

Mad2 undergoes net synthesis during meiosis I and is specifically depleted by morpholinos

Prophase I-arrested mouse oocytes, identifiable by the presence of a nucleus or germinal vesicle (GV), spontaneously resume and complete meiosis I in vitro and thereafter arrest at metaphase II. Resumption and completion of meiosis I is marked by GV breakdown (GVBD) and first polar body extrusion (PBE), respectively (Fig. 1A). In mouse oocytes from the MF1 strain, the duration of meiosis $\mathrm{I}$ as measured by the interval from GVBD to PBE lasts $\sim 11 \mathrm{~h}$ (Fig. 2A). Immunoblotting revealed that Mad2 in mouse oocytes undergoes net synthesis during progression through meiosis I (Fig. 1B, lanes 3-5), contrasting with the unchanging abundance of Mad2 reported during mitosis (Fang 2002). Relative to levels at the GV stage, Mad2 increases approximately twofold by mid-meiosis I and $\sim 10$-fold by metaphase II (Fig. 1B). Of note, metaphase II human oocytes were also found to express Mad2 (Fig. 1B, lane 2). By using a GFPtagged hMad2 construct previously shown to be a valid substitute for endogenous Mad2 in both mammalian somatic cells (Howell et al. 2000) and mouse oocytes (Wassmann et al. 2003), we estimated the concentration 
A

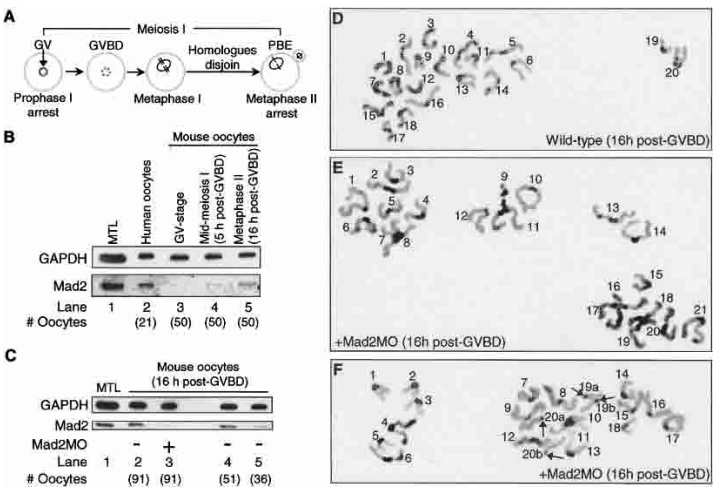

Figure 1. Depletion of Mad2 results in aneuploidy. (A) Schematic representation of meiosis I in mouse oocytes. See text for further details. (B) Mouse oocytes at the GV-stage (lane 3), at mid-meiosis I (lane 4), and at metaphase II (lane 5) were blotted for Mad2 alongside metaphase II human oocytes (lane 2). Mouse testes lysate (MTL; lane 1) and GAPDH served as positive and loading controls, respectively. $(C)$ Mouse oocytes microinjected with Mad2MO at the GV-stage were blotted for Mad2 after they had completed meiosis I. Note that Mad2 levels in 91 Mad2depleted oocytes (lane 3) are lower than levels in 51 (lane 4) and 36 (lane 5) wild-type oocytes. MTL (lane 1) and GAPDH served as positive and loading controls, respectively. $(D)$ C-banded spread of a wild-type metaphase II oocyte possessing a normal chromosome compliment of 20 monovalents numbered 1-20. (E) A typical C-banded spread of a hyperploid metaphase II oocyte resulting from $\mathrm{Mad} 2$ depletion possessing $20+$ one monovalents numbered 1-21. (F) A spread of a Mad2-depleted metaphase II oocyte displaying chromatids. Monovalents are numbered 1-18 and the four chromatids numbered 19a, 19b, 20a, and $20 \mathrm{~b}$ are indicated by arrows. The total chromosome compliment is equivalent to 20 monovalents ( 18 monovalents + four chromatids). Chromosome numbering relates to sequential order and not to karyotypic nomenclature. Original magnification, $1000 \times$.

of Mad2 to be $\sim 200 \mathrm{nM}$ by mid-meiosis I (Supplementary Fig. 1). This is similar to the concentration of Mad2 estimated to be present in HeLa cells (230 nM) (Fang 2002).

Next, we knocked down Mad2 in mouse oocytes using a morpholino oligonucleotide ( $\mathrm{MO}$ ) designated Mad2MO that was designed to target the start region of Mad2a (Supplementary Fig. 2A), the functional gene among four Mad2 genes identified in mouse (Dobles et al. 2000). Data from three separate Western blots of oocytes injected with Mad2MO and control uninjected oocytes showed that Mad2 levels were depleted by at least $85 \%$ in morpholino-injected oocytes (Fig. 1C). In contrast, two control MOs, one presenting a 5-nucleotide (nt) mispair to Mad2MO (5MispMO) and a standard control MO (StdConMO) did not affect Mad2 levels (Supplementary Fig. 2). In order to determine the efficiency of Mad2 depletion in individual oocytes, we performed immunofluorescence of uninjected control oocytes and morpholino-injected oocytes (Supplementary Fig. 3A). Consistent with immunoblotting data, the ratio of Mad2 fluorescence to background fluorescence was significantly reduced in the morpholino-injected group $(2.17 \pm 0.79$, mean $\pm \mathrm{SD} ; n=11)$ compared with uninjected controls $(6.44 \pm 1.24$, mean $\pm \mathrm{SD} ; n=13)$ but did not differ from that of oocytes exposed to secondary antibody alone
$(1.48 \pm 0.36$, mean \pm SD; $n=7)$ (Supplementary Fig. 3B) To confirm the specificity of Mad2MO, we determined whether it would target hMad2, which differs from Mad2a by $4 \mathrm{nt}$ in the target sequence (Supplementary Fig. 4A). We found that Mad2MO did not target hMad2 as oocytes coinjected with Mad2MO and hMad2-GFP mRNA expressed similar levels of hMad2-GFP to oocytes injected with hMad2-GFP mRNA alone (Supplementary Fig. 4B-E).

\section{Mad2 is required for accurate homolog disjunction}

A complete absence of Mad2 during meiosis I in yeast (Bernard et al. 2001; Shonn et al. 2000) and reduced levels of Mad2 during mammalian mitosis (Michel et al. 2001) result in chromosome missegregation. We reasoned that if Mad2 was important during female meiosis I, then depletion of Mad2 would induce aneuploidy in mouse oocytes.

To examine the impact of Mad2 depletion on homolog disjunction, we performed air-dried chromosome spreads of metaphase II oocytes that had been injected with Mad2MO at the GV-stage. Although aneuploidy can occur through loss (hypoploidy) or gain (hyperploidy) of chromosomes, we confined our analyses to the latter as other types of chromosomal aberrations can be influ-

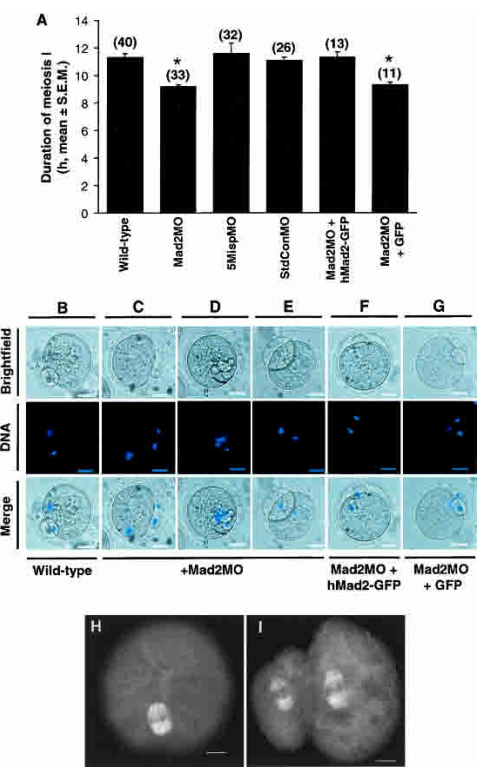

Figure 2. Effect of Mad2 depletion on the duration of meiosis I and polar body morphology. (A) Wild-type oocytes and oocytes microinjected at the GV-stage with one of either Mad2MO, 5MispMO, StdConMO, Mad2MO + hMad2-GFP mRNA, or $\mathrm{Mad} 2 \mathrm{MO}+\mathrm{GFP}$ were followed by time-lapse microscopy to measure the duration of meiosis I. Oocyte numbers from three experiments are in parentheses. Asterisks denote statistically significant differences by Student's $t$-test when compared with wild-type oocytes $(p<0.01)$. $(B-G)$ Wild-type oocytes $(B)$ or oocytes microinjected at the GV-stage with Mad2MO $(C-E)$; Mad2MO + hMad2-GFP mRNA $(F)$; or Mad2MO + GFP $(G)$ were stained for DNA (blue) after completing meiosis I. Bars, 20 $\mu \mathrm{m}$. $(H, I)$ Oocytes injected with Mad2MO at the GV-stage were immunostained for $\beta$-tubulin (white) at metaphase $\mathrm{I}(H)$ and at metaphase II $(I)$. Bars, $10 \mu \mathrm{m}$. 
enced by spreading conditions or duration in culture (Eichenlaub-Ritter and Boll 1989; Cukurcam et al. 2003). The vast majority of control oocytes had a normal chromosome compliment of 20 monovalents (Fig. 1D), with only two of $170(1.2 \%)$ being hyperploid. In contrast, following Mad2 depletion, hyperploidy was observed in 16 of $98(16.3 \%)$ oocytes. A single extra monovalent (21 monovalents in total) (Fig. 1E) was observed in 14 of 16 oocytes, while the remaining two hyperploid oocytes contained a total of 25 and 26 monovalents. Assuming an equal incidence of hypoploidy, the total incidence of aneuploidy in Mad2-depleted oocytes was therefore estimated to be $\sim 32 \%$. In all cases, hyperploidy was due to the presence of extra monovalents; no intact bivalents were observed (Fig. 1E). We were interested in determining whether Mad2-depleted oocytes exhibited premature sister chromatid separation indicative of precocious loss of centromeric cohesion. C-banding revealed that single chromatids were not a feature of aneuploid oocytes and occurred in one of $98(\sim 1 \%)$ Mad2-depleted oocytes (Fig. $1 \mathrm{~F}$, arrows) similar to the frequency of three of 170 $(1.8 \%)$ in wild-type oocytes. The data indicate that Mad2 is required for normal segregation of homologous pairs but is not required to protect centromeric cohesion in meiosis I mouse oocytes.

\section{Mad2 regulates the duration of meiosis I} and determines polar body morphology

In HeLa cells, depletion of Mad2 shortens the duration of mitosis from $\sim 100$ to $\sim 60 \mathrm{~min}$ and roughly halves the interval from nuclear envelope breakdown to anaphase, resulting in chromosome missegregation (Meraldi et al. 2004; Michel et al. 2004). We surmised that the increased aneuploidy observed in mouse oocytes following deple- tion of Mad2 might be due to accelerated progression through meiosis I. We therefore performed time-lapse imaging to determine the duration of meiosis I and found that it was $\sim 2$ h shorter in Mad2-depleted oocytes compared with wild-type or mock depleted oocytes (Fig. 2A). In addition, $\sim 60 \%$ of Mad2-depleted oocytes produced large or multiple polar bodies $(n=48)$ (Fig. 2C-E) compared with $\sim 15 \%$ of wild-type oocytes $(n=50)$ (Fig. $2 B)$. The duration of meiosis I in Mad2MO-injected oocytes could be restored to wild-type ranges by coinjecting low levels of hMad2-GFP mRNA but not by coinjecting recombinant GFP (Fig. 2A). In the presence of hMad2-GFP, $\sim 23 \%$ of Mad2MO-injected oocytes produced abnormal polar bodies $(n=13)$ (Fig. $2 F)$, whereas in the presence of GFP, aberrant PBE occurred in $\sim 55 \%$ of cases $(n=11$; Fig. 2G). This demonstrates that the effects produced by Mad2MO were specifically due to depletion of endogenous murine Mad2 $(\operatorname{mMad} 2)$ as hMad2, which is not targeted by Mad2MO (Supplementary Fig. 4), could rescue the phenotype induced by injection of Mad2MO. Abnormal polar body morphology was not due to aberrant spindle structure as Mad2-depleted oocytes assembled normal barrel-shaped bipolar spindles both at metaphase I $(n=5)$ (Fig. 2H) and at metaphase II $(n=5)$ (Fig. 2I; Supplementary Fig. 3A). Abnormal polar bodies following Mad2 knockdown were likely due to PBE occurring before the spindle had completed its migration to the oocyte cortex, which occurs during the final $2 \mathrm{~h}$ of meiosis I in mouse oocytes and contributes to polar body morphology (Verlhac et al. 2000).

\section{Mad2 depletion advances the onset of cyclin B and securin destruction}

In somatic cells, disruption of spindle checkpoint function shortens the duration of mitosis due to accelerated

Table 1. Effect of Mad2 depletion on the onset of cyclin B-GFP and securin-GFP destruction

\begin{tabular}{|c|c|c|c|c|c|c|}
\hline \multirow[b]{2}{*}{ Experiment } & \multirow[b]{2}{*}{$n$} & \multicolumn{3}{|c|}{ Onset of destruction (h post-GVBD) } & \multicolumn{2}{|c|}{ Peak fluorescence } \\
\hline & & mean \pm SEM & range & $p$ & mean \pm SEM & $p$ \\
\hline & & & & vs. $(A)$ & & vs. $(A)$ \\
\hline Cyclin B-GFP mRNA $(A)$ & 13 & $8.5 \pm 0.3$ & $6.7-10.3$ & & $305.4 \pm 36.4$ & \\
\hline Cyclin B-GFP mRNA + 5MispMO $(B)$ & 12 & $8.2 \pm 0.4$ & $6.3-10.3$ & 0.49 & $286.4 \pm 42.9$ & 0.75 \\
\hline Cyclin B-GFP mRNA + Mad2MO $(C)$ & 15 & $6.3 \pm 0.4$ & $4.3-7.7$ & 0.0004 & $270.8 \pm 33.1$ & 0.49 \\
\hline Cyclin B-GFP mRNA + AS $(D)$ & 7 & $8.5 \pm 0.5$ & $6.7-9.7$ & 0.99 & $87.1 \pm 6.7$ & 0.001 \\
\hline \multirow[t]{2}{*}{ Cyclin B-GFP mRNA + Mad2MO + AS $(E)$} & 8 & $6.1 \pm 0.3$ & $4.3-7.2$ & $<0.0001$ & $114.7 \pm 12.4$ & 0.0002 \\
\hline & & & & vs. $(F)$ & & vs. $(F)$ \\
\hline Securin-GFP mRNA $(F)$ & 11 & $8.5 \pm 0.5$ & $7.0-10.0$ & & $241.6 \pm 39.6$ & \\
\hline Securin-GFP mRNA + StdConMO $(G)$ & 13 & $8.0 \pm 0.2$ & $7.0-8.7$ & 0.24 & $259.5 \pm 21.5$ & 0.7 \\
\hline \multirow[t]{3}{*}{ Securin-GFP mRNA + Mad2MO $(H)$} & 15 & $6.9 \pm 0.2$ & $5.3-7.7$ & 0.003 & $299.7 \pm 24.4$ & 0.21 \\
\hline & & & & $(C)$ vs. $(H)$ & & $(A)$ vs. $(F)$ \\
\hline & & & & 0.14 & & 0.26 \\
\hline
\end{tabular}

Timelapse fluorescence analysis was performed on the following: (1) Oocytes microinjected with cyclin B-GFP mRNA $(A)$ or securinGFP mRNA $(F)$ only; (2) mock-depleted oocytes microinjected with cyclin B-GFP mRNA (B) or securin-GFP mRNA (G); (3) Mad2depleted oocytes microinjected with cyclin B-GFP mRNA $(C)$ or securin-GFP mRNA $(H)$; and $(4)$ wild-type $(D)$ or Mad2-depleted $(E)$ oocytes in which cyclin B-GFP levels were clamped using a GFP-targeting antisense (AS) (see Supplementary Fig. 4A). The onset of destruction was defined as the intersection between the final upward and downward phases of the cyclin B-GFP or securin-GFP time-lapse profiles as illustrated in Figure 3F. Statistical analysis by Student's $t$-test. 

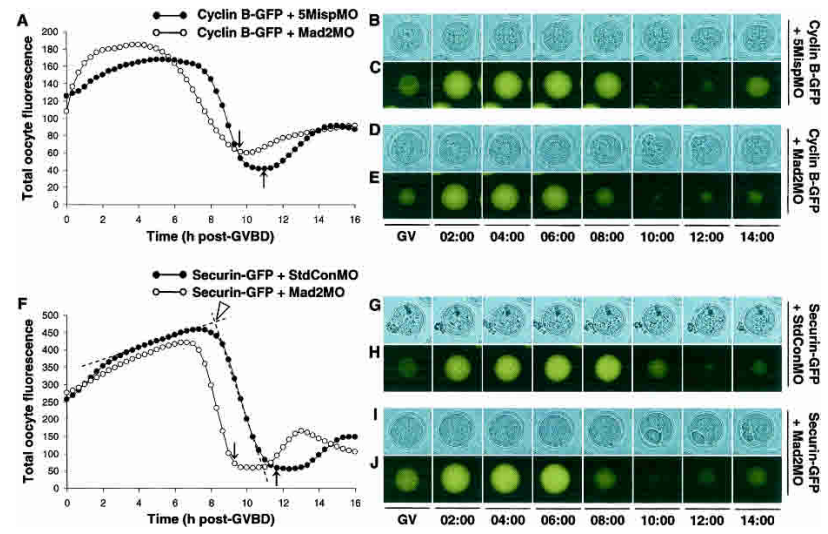

Figure 3. Depletion of Mad2 advances the onset of cyclin B and securin destruction. $(A-E) \mathrm{GV}$-stage oocytes coinjected with cyclin B-GFP mRNA and either Mad2MO or 5MispMO were analyzed by time-lapse microscopy during progression through meiosis I. $(F-J) \mathrm{GV}$-stage oocytes coinjected with securin-GFP mRNA and either Mad2MO or StdConMO were examined by time-lapse microscopy during progression through meiosis I. Shown are profiles of representative oocytes $(A, F)$ and their corresponding brightfield $(B, D, G, I)$ and fluorescence frames $(C, E, H, J)$ at the indicated times post-GVBD in hours:minutes. $(A, F)$ Black arrows indicate the times of PBE. $(F)$ Open arrowhead denotes the intersection between the final upward and downward phases (dashed lines) of a representative securin-GFP profile illustrating the method used for determining the time of onset of protein destruction for the data displayed in Table 1. Note the multiple polar bodies in $I$. Bars, $20 \mu \mathrm{m}$.

anaphase onset (Meraldi et al. 2004), which in turn is mirrored by an earlier onset of cyclin B and securin destruction (Hagting et al. 2002; Michel et al. 2004). In order to determine whether this was also the case in meiosis I, we monitored the timing of degradation of cyclin B and securin using time-lapse fluorescence imaging of GFP-tagged chimerae, which are valid markers for their endogenous counterparts and are destroyed in a checkpoint-dependent manner in mammalian cells (Clute and Pines 1999; Hagting et al. 2002). In addition, these GFP constructs do not affect progression through meiosis I in mouse oocytes when expressed at low levels (Herbert et al. 2003). Wild-type and mock depleted oocytes readily synthesized cyclin B-GFP and securin-GFP from microinjected mRNAs and commenced their destruction from 8 to $9 \mathrm{~h}$ post-GVBD (Table 1; Fig. 3A-C,F-H). Following Mad2 depletion, however, destruction occurred significantly earlier $(6-7 \mathrm{~h}$ postGVBD) for both cyclin B-GFP and securin-GFP (Table 1; Fig. 3A,D-F,IJJ). These differences were not due to differences in cyclin B-GFP or securin-GFP expression, as peak fluorescence intensities did not differ between test and control groups (Table 1). To examine this further, we used an antisense designed to target GFP, which arrests translation from cyclin B-GFP mRNA in mouse oocytes (Nixon et al. 2002). Injecting oocytes with GFP-targeting antisense $\sim 2 \mathrm{~h}$ following injection with cyclin B-GFP mRNA (Supplementary Fig. 5A) clamped cyclin B-GFP levels at $\sim 30 \%$ of the peak levels attained with mRNA alone (Table 1). Even with these reduced levels of expression, cyclin B destruction still occurred $\sim 2 \mathrm{~h}$ earlier following Mad2 knockdown (Table 1; Supplementary Fig. 5B-F).
These findings indicate that the shortened duration of meiosis I following Mad2 depletion (Fig. 2A) is due to advanced anaphase I onset. Furthermore, the regulation of cyclin B and securin proteolysis by Mad2 is confined to $\mathrm{a} \sim 2 \mathrm{~h}$ window during the final stages of meiosis $\mathrm{I}$. Stable kinetochore-microtubule interactions are established in mouse oocytes towards the end of meiosis I (Brunet et al. 1999). By deregulating the final phases of meiosis I, Mad2 depletion is therefore likely to disrupt the process of kinetochore-microtubule interaction, thereby predisposing to nondisjunction.

\section{Mad2 overexpression inhibits homolog disjunction}

In somatic cells and meiosis I mouse oocytes, overexpression of Mad2 induces a metaphase arrest (Howell et al. 2000; Wassmann et al. 2003). The sensitivity of somatic cells to metaphase arrest is graded so that $\sim 10$-fold overexpression of Mad2 above endogenous levels induces a metaphase arrest, whereas at lower levels of overexpression (approximately twofold excess) mitosis proceeds with normal kinetics (Howell et al. 2000). The sensitivity of mouse oocytes to Mad2 overexpression could not be ascertained from previous data, as levels of exogenous hMad2 were not quantified (Wassmann et al. 2003). To address this, we injected oocytes with hMad2GFP mRNA and quantified expression levels using a calibration curve generated by injecting oocytes with known concentrations of recombinant GFP (Supplementary Fig. 1A). Oocytes expressing a fourfold excess of hMad2-GFP over endogenous mMad2 underwent PBE in $\sim 75 \%$ of cases, similar to wild-type oocytes (Fig. 4A). At 15-fold excess levels of hMad2-GFP, however, there was a significant inhibition of $\mathrm{PBE}$, and above 35 -fold excess

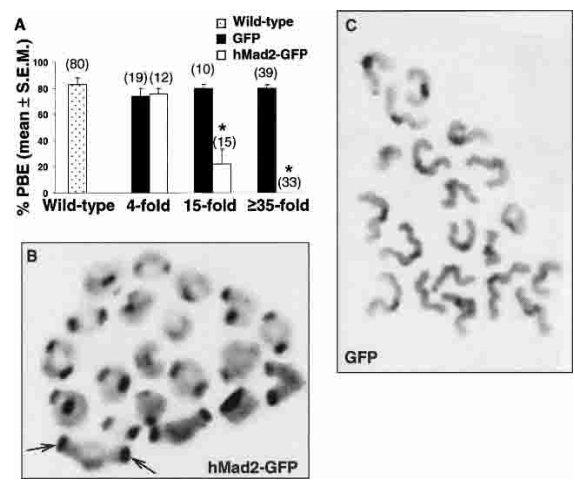

Figure 4. Overexpression of hMad2-GFP inhibits homolog disjunction. (A) Wild-type oocytes or GV-stage oocytes microinjected with either hMad2-GFP mRNA or recombinant GFP were cultured in vitro and examined for $\mathrm{PBE}$ at $16 \mathrm{~h}$ post-GVBD. Intracellular concentrations of hMad2-GFP and GFP were estimated utilizing a calibration curve (see Supplementary Fig. 1A) and expressed as fold excess over endogenous mMad2. Oocyte numbers from four experiments are in parentheses. Asterisks denote statistically significant differences by Student's $t$-test when compared with wild-type or GFP-injected oocytes $(p<0.0001) .(B, C)$ C-banded chromosome spreads performed at $16 \mathrm{~h}$ post-GVBD of oocytes microinjected at the GV-stage with either hMad2-GFP mRNA $(B)$ or recombinant GFP $(C)$. Intact bivalents possess darkly-staining centromeres at opposing ends (e.g., arrows in $B$ ) as opposed to a central location in monovalents $(C)$. Original magnification, 1000x. 
levels PBE was completely inhibited, whereas similar levels of GFP alone did not affect meiosis I progression (Fig. 4A). Oocytes arrested by hMad2-GFP were at metaphase I (data not shown) and possessed intact bivalents (Fig. 4B), whereas in the presence of GFP alone homolog disjunction was unaffected (Fig. 4C). Thus, Mad2 inhibits homolog disjunction in mouse oocytes when overexpressed by a similar order of magnitude to that which induces a mitotic arrest (Howell et al. 2000). Notably, lower levels of hMad2-GFP did not affect the duration of meiosis I since mRNA-injected oocytes that completed meiosis I underwent PBE at a similar time to wild-type oocytes (Supplementary Fig. 6). Thus, in the presence of exogenous Mad2, meiosis I is either arrested or proceeds at a normal rate.

\section{Conclusions}

Here we show that Mad2 is critical for accurate homolog disjunction and for the temporal regulation of cyclin $\mathrm{B}$ and securin degradation during meiosis I in mouse oocytes in the absence of exogenous damage. Given that the APC/C ${ }^{\mathrm{Cdc} 20}$ is currently the only known target of the Mad2-dependent mitotic spindle checkpoint, these data also suggest that proteolysis of cyclin B and securin is mediated by the APC/C $\mathrm{C}^{\mathrm{Cdc} 20}$ in mammalian oocytes. In support of this, we have shown that cyclin B and securin degradation are entirely dependent upon the destruction box (Herbert et al. 2003), the APC/ $\mathrm{C}^{\mathrm{Cdc} 20}$ substrate recognition motif (Peters 2002).

During budding yeast meiosis I, Mad2 actively participates in establishing bipolar oriented bivalents (Shonn et al. 2003). Following loss of Mad2, therefore, mono-oriented bivalents persist, resulting in migration of both members of a homologous pair to one pole at anaphase I (Shonn et al. 2003). It remains to be established whether Mad2 fulfils a similar function during meiosis I in mouse oocytes, but such a role would be consistent with the pattern of hyperploidy (extra monovalents) that we observed following Mad2 depletion. Furthermore, a lack of prematurely separated sister chromatids in Mad2-depleted oocytes indicates that centromeric cohesion was maintained, consistent with findings in fission yeast (Bernard et al. 2001). Of note, it has recently been shown that depletion of Mad2 accelerates mitotic progression through loss of a "timing" function, which is quite independent of Mad2's classical checkpoint role (Meraldi et al. 2004). The $\sim 2$-h-shorter duration of meiosis I following Mad2 depletion (Fig. 2A) may reflect a similar timing function for Mad2 in female meiosis I. Loss of Mad2 might therefore disrupt the fidelity of homolog disjunction on a number of different levels: Not only is the machinery for detecting and correcting mal-oriented chromosomes handicapped but the time available for spontaneously righting them is markedly reduced.

Our results demonstrate that Mad2 depletion results in extra monovalents without an increase in single chromatids. Although earlier reports of metaphase II human oocytes suggest that the majority of human aneuploidies are due to premature separation of sister chromatids (Angell et al. 1993), more recent observations in fresh human oocytes indicate that nondisjunction of whole monovalents also occurs (Sandalinas et al. 2002). It is conceivable that impaired Mad2 function could contribute to nondisjunction-type errors and that defects in other proteins such as Sgol, a conserved protein that protects centromeric cohesion during anaphase I of fission yeast (Kitajima et al. 2004; Rabitsch et al. 2004), could contribute to premature sister chromatid separation, together producing a mixed pattern of aneuploidy.

While our findings are consistent with yeast meiosis I (Bernard et al. 2001; Shonn et al. 2000), they appear to be at odds with previous meiosis I studies in mouse oocytes conducted in the absence of microtubule inhibitors. First, it was recently reported that a dominant-negative hMad2 had no effect on meiosis I in mouse oocytes in the absence of spindle poisons (Wassmann et al. 2003), whereas our data indicate that $\geq 85 \%$ depletion of $\operatorname{Mad} 2$ does. Mitosis may provide a precedent for explaining this apparent paradox. Following 30\% reduction in Mad2 levels, HeLa cells fail to arrest in the presence of nocodazole and prematurely inactivate Cdk1 (Michel et al. 2001), yet there are no overt effects on mitosis in unperturbed cells in terms of spindle assembly and cell-cycle progression (Michel et al. 2004). Following 90\% Mad2 depletion, however, spindle assembly and chromosome condensation are grossly perturbed, cyclin B is degraded in early prometaphase, and mitosis is shortened by $\sim 40 \%$ (Michel et al. 2004). $\mathrm{PtK}_{1}$ cells injected with Mad2 antibodies initiate anaphase prematurely (Gorbsky et al. 1998) but assemble normal spindles, suggesting that a substantial level of Mad2 function remains (see Michel et al. 2004). It may be that like mitosis, mouse oocytes demonstrate a graded response to differing degrees of loss of Mad2 function. Thus, the dominant-negative hMad2 may have moderately disrupted mMad2 function sufficient to destabilize Cdk1 in the presence of nocodazole but not to an extent that would produce overt effects on meiosis I (Wassmann et al. 2003), such effects becoming more apparent following $\geq 85 \%$ Mad2 depletion (data not shown). Second, on the basis of evidence from mouse oocytes monosomic for the X chromosome (XO oocytes), it has been argued that female mammalian meiosis I lacks a spindle checkpoint (LeMaire-Adkins et al. 1997). $\mathrm{XO}$ oocytes fail to delay anaphase I despite the presence of the misaligned $\mathrm{X}$ univalent, which is frequently accompanied by a small number of misaligned autosomes (LeMaire-Adkins et al. 1997). In contrast, anaphase and exit from meiosis I are inhibited in oocytes of $\mathrm{Mlh1}^{-/-}$ mice, which exhibit dramatically reduced recombination and gross misalignment of univalents at meiosis I (Woods et al. 1999). Together these data raise the possibility that the oocyte spindle checkpoint is not sufficiently sensitive to detect a small number of misaligned chromosomes. Alternatively, in view of the accelerated progression of XO oocytes through meiosis I (LeMaireAdkins et al. 1997), reminiscent of the phenotype observed following Mad2 depletion, it would be interesting to test the idea that XO oocytes have an inherent checkpoint defect, which not only renders them insensitive to the misaligned $X$ univalent but also allows insufficient time for stable biorientation of chromosomes. In this respect, it is tempting to speculate that the long duration of meiosis I in mammalian oocytes reduces the risk of aneuploidy by giving bivalents more time to biorientate.

It has been proposed that a decline in spindle checkpoint function contributes to Down syndrome (Shonn et al. 2003). Our data are consistent with this and imply that such a decline may be a consequence of reduced expression of checkpoint proteins. In support of a link between Mad2 levels and the increased incidence of trisomic pregnancy in reproductively aged women (Hassold 
and Hunt 2001), it has been reported that oocytes of older women exhibit a trend toward lower levels of Mad2 mRNA transcripts compared with those of younger women (Steuerwald et al. 2001). Furthermore, in mice, maternal aging is associated with accelerated progression through meiosis I and an increased incidence of aneuploidy (Eichenlaub-Ritter and Boll 1989), which our data indicate are both consequences of reduced Mad2 levels. Thus, the culmination of evidence suggests that reduced expression of checkpoint proteins contributes to the maternal age-related increase in aneuploidies of meiosis I origin.

\section{Materials and methods}

GFP constructs, morpholinos, and GFP antisense

Capped hMad2-GFP mRNA was produced by subcloning the full-length sequence for hMad2 into the pMDL2 transcription vector followed by in vitro transcription as described (Levasseur and McDougall 2000). Human cyclin B1-GFP and securin-GFP constructs have been described (Herbert et al. 2003). Constructs were injected at a concentration of $0.25-1 \mu \mathrm{g} / \mu \mathrm{L}$.

Morpholino sequences (Gene Tools LLC) were injected at a concentration of $17 \mu \mathrm{g} / \mu \mathrm{L}$. The GFP-targeting antisense (Nixon et al. 2002) was injected at a concentration of $0.5 \mu \mathrm{g} / \mu \mathrm{L}$.

\section{Air-dried chromosome spreads and C-banding}

Chromosome preparations and C-banding were performed as described previously (Eichenlaub-Ritter and Boll 1989). To eliminate overlapping of spreads, oocytes were individually pipetted on to premarked positions on slides. For each oocyte, the number of chromosomes was counted under phase-contrast using a Nikon Eclipse E400 inverted microscope. Oocytes were excluded from the analysis based on the following criteria: inadequate C-banding for discriminating chromosome morphology, overlapping or clustering of chromosomes, or excessive chromosome scatter.

\section{Acknowledgments}

We thank J. Pines, J. Pinto-Toro, and R. Benezra for the generous gifts of plasmids. We acknowledge K. Lacsko and all members of the laboratory for technical assistance and advice. This work was supported by a WellBeing Research Training Fellowship (RTF/387) to H.A.H and grants from Newcastle University Hospitals Special Trustees and Wellcome to M.H. and A.M.D.

\section{References}

Angell, A.A., Xian, J., and Keith, J. 1993. Chromosome anomalies in human oocytes in relation to age. Hum. Reprod. 8: 1047-1054.

Bernard, P., Maure, J.F., and Javerzat, J.P. 2001. Fission yeast Bubl is essential in setting up the meiotic pattern of chromosome segregation. Nat. Cell Biol. 3: 522-526.

Brunet, S., Santa Maria, A., Guillaud, P., Dujardin, D., Kubiak, J.Z., and Maro, B. 1999. Kinetochore fibers are not involved in the formation of the first meiotic spindle of mouse oocytes, but control the exit from the first meiotic M phase. J. Cell. Biol. 146: 1-11.

Clute, P. and Pines, J. 1999. Temporal and spatial control of cyclin B1 destruction in metaphase. Nat. Cell Biol. 1: 82-87.

Cukurcam, S., Hegele-Hartung, C., and Eichenlaub-Ritter, U. 2003. Meiosis-activating sterol protects oocytes from precocious chromosome segregation. Hum. Reprod. 18: 1908-1917.

Dobles, M., Liberal, V., Scott, M.L., Benezra, R., and Sorger, P.K. 2000. Chromosome missegregation and apoptosis in mice lacking the mitotic checkpoint protein Mad2. Cell 101: 635-645.

Eichenlaub-Ritter, U. and Boll, I. 1989. Nocodazole sensitivity, age-related aneuploidy, and alterations in the cell cycle during maturation of mouse oocytes. Cytogent. Cell Genet. 52: 170-176.

Fang, G. 2002. Checkpoint protein BubR1 acts synergistically with Mad2 to inhibit anaphase-promoting complex. Mol. Biol. Cell 13: 755-766.

Gorbsky, G.J., Chen, R.H., and Murray, A.W. 1998. Microinjection of antibody to Mad2 protein into mammalian cell in mitosis induces premature anaphase. J. Cell. Biol. 141: 1193-1205.
Hagting, A., den Elzen, N., Vodermaier, H.C., Waizenegger, I.C., Peters, J.M., and Pines, J. 2002. Human securin proteolysis is controlled by the spindle checkpoint and reveals when the APC/C switches from activation by Cdc20 to Cdh1. J. Cell. Biol. 157: 1125-1137.

Hassold, T. and Hunt, P. 2001. To err (meiotically) is human: The genesis of human aneuploidy. Nat. Rev. Genet. 2: 280-291.

Herbert, M., Levasseur, M., Homer, H., Yallop, K., Murdoch, A., and McDougall, A. 2003. Homologue disjunction in mouse oocytes requires proteolysis of securin and cyclin B1. Nat. Cell Biol. 5: 10231025.

Howell, B.J., Hoffman, D.B., Fang, G., Murray, A.W., and Salmon, E.D. 2000. Visualization of Mad2 dynamics at kinetochores, along spindle fibers, and at spindle poles in living cells. J. Cell. Biol. 150: 12331249.

Kalitsis, P., Earle, E., Fowler, K.J., and Andy Choo, K.H. 2000. Bub3 gene disruption in mice reveals essential mitotic spindle checkpoint function during early embryogenesis. Genes \& Dev. 14: 2277-2282.

Kitajima, T.S., Kawashima, S.A., and Watanabe, Y. 2004. The conserved kinetochore protein shugoshin protects centromeric cohesion during meiosis. Nature 427: 510-517.

LeMaire-Adkins, R., Radke, K., and Hunt, P.A. 1997. Lack of checkpoint control at the metaphase/anaphase transition: A mechanism of meiotic nondisjunction in mammalian females. J. Cell. Biol. 139: 16111619.

Levasseur, M. and McDougall, A. 2000. Sperm-induced calcium oscillations at fertilisation in ascidians are controlled by cyclin B1-dependent kinase activity. Development 127: 631-641.

Li, Y. and Benezra, R. 1996. Identification of a human mitotic checkpoint gene: hsMAD2. Science 274: 246-248.

Meraldi, P., Draviam, V.M., and Sorger, P.K. 2004. Timing and checkpoints in the regulation of mitotic progression. Dev. Cell 7: 45-60.

Michel, L.S., Liberal, V., Chatterjee, A., Kirchwegger, R., Pasche, B., Gerald, W., Dobles, M., Sorger, P.K., Murty, V.V., and Benezra, R. 2001. MAD2 haplo- insufficiency causes premature anaphase and chromosome instability in mammalian cells. Nature 409: 355-359.

Michel, L., Diaz-Rodriguez, E., Narayan, G., Hernando, E., Murty, V.V.V.S., and Benezra, R. 2004. Complete loss of the tumor suppressor MAD2 causes premature cyclin B degradation and mitotic failure in human somatic cells. Proc. Natl. Acad. Sci. 101: 4459-4464.

Mussachio, A. and Hardwick, K. 2002. The spindle checkpoint: Structural insights into dynamic signalling. Nat. Rev. Mol. Cell Biol. 3: 731-741.

Nixon, V.L., Levasseur, M., McDougall, A, and Jones, K.T. 2002. $\mathrm{Ca}^{2+}$ oscillations promote APC/C-dependent cyclin B1 degradation during metaphase arrest and completion of meiosis in fertilizing mouse eggs. Curr. Biol. 12: 746-750.

Peters, J.M. 2002. The anaphase-promoting complex: Proteolysis in mitosis and beyond. Mol. Cell 9: 931-943.

Rabitsch, K.P., Gregan, J., Schleiffer, A., Javerzat, J.P., Eisenhaber, F., and Nasmyth, K. 2004. Two fission yeast homologs of Drosophila MeiS332 are required for chromosome segregation during meiosis I and II. Curr. Biol. 14: 287-301.

Sandalinas, M., Marquez, C., and Munne, S. 2002. Spectral karyotyping of fresh, noninseminated oocytes. Mol. Hum. Reprod. 8: 580-585.

Shonn, M.A., McCarroll, R., and Murray, A.W. 2000. Requirement of the spindle checkpoint for proper chromosome segregation in budding yeast meiosis. Science 289: 300-303.

Shonn, M.A., Murray, A.L., and Murray, A.W. 2003. Spindle checkpoint component Mad2 contributes to biorientation of homologous chromosomes. Curr. Biol. 13: 1979-1984.

Steuerwald, N., Cohen, J., Herera, R.J., Sandalinas, M., and Brenner, C.A. 2001. Association between spindle assembly checkpoint expression and maternal age in human oocytes. Mol. Hum. Reprod. 7: 49-55.

Verlhac, M., Lefebvre, C., Guillaud, P., Rassinier, P., and Maro, B. 2000. Asymmetric division in mouse oocytes with or without Mos. Curr. Biol. 10: 1303-1306.

Wassmann, K., Niault, T., and Maro, B. 2003. Metaphase I arrest upon activation of the MAD2-dependent spindle checkpoint in mouse oocytes. Curr. Biol. 13: 1596-1608.

Woods, L.M., Hodges, C.A., Baart, E., Baker, S.M., Liskay, M., and Hunt, P.A. 1999. Chromosomal influence on meiotic spindle assembly: Abnormal meiosis I in female Mlh1 mutant mice. J. Cell. Biol. 145: $1395-1406$. 


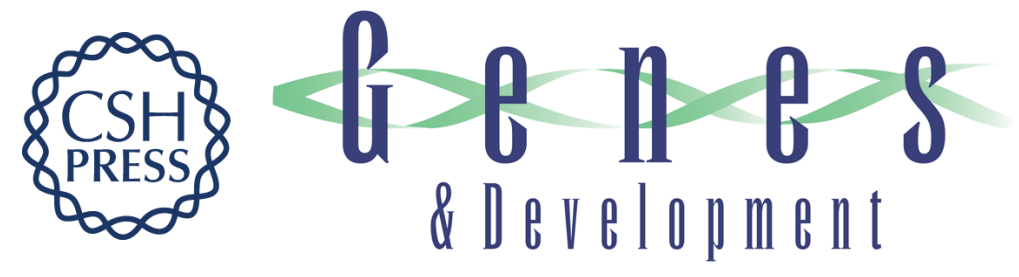

\section{Mad2 prevents aneuploidy and premature proteolysis of cyclin B and securin during meiosis I in mouse oocytes}

Hayden A. Homer, Alex McDougall, Mark Levasseur, et al.

Genes Dev. 2005, 19:

Access the most recent version at doi:10.1101/gad.328105

\section{Supplemental http://genesdev.cshlp.org/content/suppl/2004/12/29/19.2.202.DC1 Material}

References This article cites 32 articles, 12 of which can be accessed free at: http://genesdev.cshlp.org/content/19/2/202.full.html\#ref-list-1

\section{License}

Email Alerting

Receive free email alerts when new articles cite this article - sign up in the box at the top Service

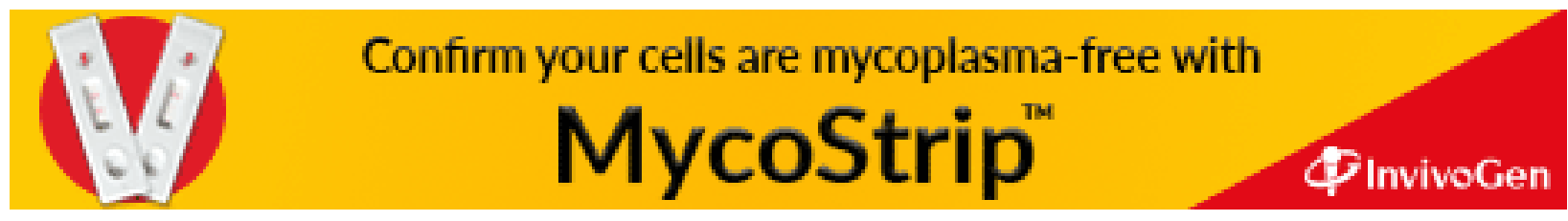

